\title{
Self-referrals to a community psychiatric clinic
}

\author{
A. P. Boardman, Lecturer in Psychiatry, National Unit for Psychiatric Research \\ and Development, Lewisham Hospital, London SE13 6LH (correspondence) and \\ Honorary Senior Registrar, Division of Psychiatry, UMDS, Guy's Hospital; and \\ N. Bouras, Consultant Psychiatrist and Honorary Lecturer, Division of Psychiatry, \\ UMDS, Guy's Hospital, London SE1
}

Over recent years a number of non-hospital based services have been developed that can be subsumed under the title of Community Mental Health Centres (CMHCs). Conferences held by the King's Fund (McAusland, 1985) and the National Unit for psychiatric Research and Development (Sayce, 1987) have highlighted the importance of CMHC-like services in health authorities' plans to develop community based psychiatric services. There is no single view of CMHCs in the United Kingdom and, while many such centres already exist, they are diverse in nature and their activities have been sparsely documented.

An issue often raised by proponents of these services is whether to allow potential patients to by-pass the normal referral channels and present themselves directly to the service. The notion of self-referrals to secondary services is a source of anxiety among psychiatrists who fear an overwhelming demand on services by an inappropriate clientele. However, there has been few documented experiences of self referrals to psychiatric services in the UK, exceptions being the Maudsley Emergency Clinic (e.g. Lim, 1983) and the Eastgate Community Mental Health Centre in Lewes (Hutton, 1985).

Hutton's account of the Eastgate CMHC was hampered by sparsity of cases and the inability to compare the self-referrals to another group. The present paper attempts to rectify this by reporting on the details of over 400 self-referrals to the Mental Health Advice Centre(MHAC) in Lewisham, South-east London.

\section{The Mental Health Advice Centre}

The Mental Health Advice Centre (MHAC) is one of the longest running CMHCs in the UK and was established in November 1978 in the geographically defined catchment area of one consultant psychiatrist responsible for patients in the north of Lewisham and is part of the psychiatric services provided by the Lewisham and North Southwark Health Authority. The centre is staffed by a multiprofessional team (MPT) who run a 'walk-in' facility for which one professional is on duty each weekday morning from 9.00 a.m. to 1.00 p.m. to see referrals. Referrals are accepted from local GPs, social workers, health visitors and other community agents, or clients can refer themselves.

Within the NHS the majority of patients are filtered by the general practitioner to secondary services (Goldberg \& Huxley, 1980). The MPT allows patients to bypass the GP and come directly to the centre. This raises the question of what differences exist between those patients who refer themselves directly to the MPT and those who are filtered by their GP. The aim of this paper is to describe the selfreferred patients to the MPT between November 1978 and the end of December 1985 and to compare these to GP referrals over the same period of time.

\section{The study}

The use of the centre has been routinely monitored since its inception and has been extensively reported (e.g. Boardman \& Bouras, 1988). Information on all patients seen by the MPT was recorded by the professional seeing the patient. Each patient was discussed at the weekly case conference, a diagnosis made by the consultant psychiatrist on the basis of written reports, and a management decision made. All the information was stored and analysed in computer form. A comparison of GP and self-referrals has been made by chi-square analysis.

\section{Total referrals}

Between November 1978 and the end of December 1985,2263 referrals were seen by the MPT of which $1863(82.3 \%)$ were new referrals and $400(17.7 \%)$ were re-referrals. A 'new' referral is a patient who has never previously been seen by the MPT, a 're-referral' is one who has been previously seen by the MPT and whose case was formally closed.

Of the new referrals, $70.2 \%$ were made by GPs and $15.7 \%$ were self referrals. For new referrals these two sources have, over time, complemented each other with the decline in the proportion of GP referrals being matched by an increase in the proportion of self-referrals. The proportion of new self-referrals 
has shown a steady increase from $4.2 \%$ in 1979 to $23.3 \%$ in 1984 and $18.4 \%$ in 1985 .

The source of the re-referrals provides a contrast to this with the majority $(62.2 \%)$ of re-referrals being self-referred and $30.3 \%$ referred by GPs $(64.5 \%$ of the self-referrals who re-referred themselves were first introduced to the service by their GP).

The MPT was set up as an ease of access service emphasising liaison with general practitioners, rapid referral by community agents, and self-referral. While this has resulted in an overall expansion of the ambulatory sector of the catchment area services (Boardman \& Bouras, 1988), it has not caused an influx of inappropriate self-referrals and the vast majority are still being filtered by their GP. The practice of allowing self-referrals has, however, meant that patients, once familiar with the service, can reestablish contact with the MPT in times of distress. Interpretation of the relevance of this group of self re-referrals is not straightforward as it could be seen as a failure of the service to provide adequate follow-up for the patients. While some of the patients were still in contact with some part of the catchment area service the majority were not and almost one quarter presented with short-lived crises (adjustment reaction). A relatively small number were multiple re-referrals $(16.9 \%$ were rereferred twice, $7.1 \%$ had referred themselves more than twice). This small number of multiple rereferrers were well known to the service as requiring a high input for longstanding and difficult problems.

\section{Demographic characteristics}

Comparison of the self-referrals with the GP referrals shows some discrete differences with regard to these two groups. There were no differences as regard to age, sex, marital status, national origin and employment status. For both the new and re-referrals there were significantly more men than women amongst the self than the GP referrals (New referrals $-\chi^{2}=3.894, \mathrm{df}=1, P<0.05$; Re-referrals $\left.\chi^{2}=5.559, \mathrm{df}=1, P<0.025\right)$. There was a significant excess of social classes $1 / I 1$ among the new selfreferrals $\left(\chi^{2}=7.823, \mathrm{df}=2, P<0.025\right)$ and the same trend was seen among the re-referrals.

Two studies (Lim, 1983; Hutton, 1985) have reported an absolute excess of men among their selfreferrals, but it is not clear from either study whether this was a general or local effect. For example, in Lim's sample the excess of men could have been accounted for by the fact that many of the selfreferred males came from local hostels for homeless men. The present figures did not show an absolute excess of males. This finding may reflect a willingness for men to present themselves directly for treatment. However, the same relative excess of men is seen among the GP referrals to the catchment area outpatient service (Boardman - unpublished data). The lower proportion of men in the GP referrals to the MPT may therefore reflect the referral preference of the GPs, i.e. they are more likely to send women to the MPT rather than the OPC.

The finding that individuals of higher social class tend to by-pass the GP has not previously been reported in the UK and may reflect the tendency of the middle classes, found in primary care studies, to initiate consultations with doctors and to take up preventive services (Crombie, 1987).

\section{Clinical characteristics}

New GP referrals were less likely than new selfreferrals to have received psychiatric care in the past, this difference almost reaching significance $\left(\chi^{2}=3.221\right.$, df $\left.=1, P<0.10\right)$, suggesting that it is those patients who have had past experience of psychiatric services that by-pass the GP. New selfreferrals are more likely to be given a diagnosis of adjustment reaction or no psychiatric diagnosis, and less likely to be given an 'other' diagnosis $\left(\chi^{2}=\right.$ 19.37, $\mathrm{df}=6, P<0.01)$. No such differences were seen among the re-referrals. The excess of these 'other' diagnoses among the new GP referrals and the lower proportion of adjustment reactions may reflect the perceived need for help among the selfreferrals who are more likely to present directly to the service with their affective symptoms. Among the 'other' category are many people with conditions (e.g. psychosomatic disorders, eating disorders) whose need for treatment may be perceived by the GP rather than the patient.

\section{Management decisions}

A decision of 'no further action' was more likely to be made for new self-referrals than for new GP referrals $\left(\chi^{2}=14.66, \mathrm{df}=7, P<0.05\right)$. This was the same for the re-referrals $\left(\chi^{2}=12.99, \mathrm{df}=6\right.$, $P<0.05)$. For the new referrals the GP referrals were more likely to be given 'other' management than the self referrals and the GP re-referrals were more likely to be admitted.

The different management decisions made for the GP and self referrals may reflect the different requests made by the referral sources, i.e. the GPs make more specific requests than the self referrals. For example, among the re-referred patients: $20.7 \%$ of the selfreferrals received 'no further action' as opposed to $9.5 \%$ of the GP referrals (this may also reflect the fact that many of the self-referrals are in contact with some part of the catchment area service), in contrast $39.2 \%$ of the GP referrals receive counselling and $10.1 \%$ are admitted as compared to $32.7 \%$ and $4.0 \%$ of self-referrals. 


\section{Comment}

The results have both service and theoretical importance. In terms of service developments there have been few reports of self-referrals to psychiatric services in the UK. The opening up of secondary services to self-referrals is a source of anxiety and uniformed speculation. The availability of data on service usage provides a basis from which to inform those involved in planning.

In addition, the differences found between GP and self-referrals allow some speculations to be made about the determinants of self-referral. It must be noted that the data presented have compared one set of secondary service users to another, the difference between these sets being that one has by-passed while the other has been filtered by the GP. This means that the comparisons can show only relative differences, thus making interpretation difficult. They do however, point to two areas of potential interest: sex and social class. While there is a wealth of data published on the use of general practice services by different demographic groups (e.g. Crombie, 1987; Collins \& Klein, 1980), little is known about self-referral to secondary services in the UK. From a theoretical perspective the data presented here provide indicators on which hypotheses and future studies can be based. In this way the monitoring and evaluation of services can be used, not only to provide an overview of service usage but also to promote theoretical investigation.

\section{Acknowledgements}

We would like to thank John Cundy for his assistance with the computer analysis. The study was supported by a grant from the Sainsbury Family Charitable Foundation.

\section{References}

Boardman, A. P. \& Bouras, N. (1988) The Mental Health Advice Centre in Lewisham. Health Trends, 20, 59-63.

Collins, E. \& KLEN, R. (1980) Equity and the NHS: selfreported morbidity, access, and primary care. British Medical Journal, 281, 1111-1115.

Crombre, D. L. (1987) Social Class and Health Status. Inequality or Difference? Royal College of General Practitioners Occasional Paper 25. Exeter: RCGP.

GOLDBerg, D. \& HuXuEY, P. (1980) Mental Illness in the Community. The Pathway to Psychiatric Care. London: Tavistock.

Hutron, H. (1985) Self-referrals to a community mental health centre: A three year study. British Journal of Psychiatry. 147, 540-544.

Lm, M. H. (1983) A psychiatric emergency clinic: A study of attendences over six months. British Journal of Psychiatry, 143, 460-466.

MCAusland, T. (1985) Planning and Monitoring Community Mental Health Centres. London: Kings Fund Centre.

SAYCE, E. (1987) Community Mental Health Centres Report of the Annual Conference 1987. London: NUPRD.

Details of the data are available from the authors on request.

\section{Quality of life in a residential setting}

\section{IAN Leonard, Research Registrar in Psychiatry, The David Lewis Centre for Epilepsy, Near Alderley Edge, Cheshire SK9 7UD}

The David Lewis Centre for Epilepsy has approximately 300 adult residents. All have epilepsy, which is often only partly alleviated by available treatments; many have mental handicap or psychiatric illness, adding to their total disability. The Centre's explicit aim of understanding and caring for people with epilepsy implies concern not just for clinical measures such as seizure frequency, but for our residents' quality of life.

Two trends in patient management over recent years combine to focus interest on quality of life measurement in residential settings. One is the shift to community from residential care, too often with an untested assumption of the former's intrinsic super- iority. The other is the increasing recognition of the necessity of service evaluation to ensure the optimal use of finite resources and justify the continuation of those resources.

While the need for evaluation of quality of life in institutions is clear, the form that evaluation should take is not. Symptoms and their alleviation seem relevant, yet this is insufficient when institutions impinge on all areas of their residents' lives. Their physical surroundings, activities through the day and social milieu are just a few examples of spheres where such influence is marked. Day \& Klein (1987) reviewed the assessment of quality of care and suggested that in the difficult area of outcome 